\title{
Order
}

\section{AZ-identities and Strict 2-part Sperner Properties of Product Posets --Manuscript Draft--}

\begin{tabular}{|c|c|}
\hline \multicolumn{2}{|l|}{ Manuscript Number: } \\
\hline Full Title: & AZ-identities and Strict 2-part Sperner Properties of Product Posets \\
\hline Article Type: & Original Research \\
\hline Keywords: & $\begin{array}{l}\text { Sperner property; strict Sperner property; strict BLYM inequality; AZ--identity; 2-part } \\
\text { Sperner property; normal poset }\end{array}$ \\
\hline Corresponding Author: & $\begin{array}{l}\text { Peter L. Erdos, Ph.D. } \\
\text { A. Renyi Institute of Mathematics } \\
\text { Budapest, n/a HUNGARY }\end{array}$ \\
\hline \multicolumn{2}{|l|}{$\begin{array}{l}\text { Corresponding Author Secondary } \\
\text { Information: }\end{array}$} \\
\hline Corresponding Author's Institution: & A. Renyi Institute of Mathematics \\
\hline \multicolumn{2}{|l|}{$\begin{array}{l}\text { Corresponding Author's Secondary } \\
\text { Institution: }\end{array}$} \\
\hline First Author: & Peter L. Erdos, Ph.D. \\
\hline \multicolumn{2}{|l|}{ First Author Secondary Information: } \\
\hline \multirow[t]{2}{*}{ Order of Authors: } & Peter L. Erdos, Ph.D. \\
\hline & Harout Aydinian, Ph.D. \\
\hline \multicolumn{2}{|c|}{ Order of Authors Secondary Information: } \\
\hline Abstract: & $\begin{array}{l}\text { One of central issues in extremal set theory is Sperner's theorem and its } \\
\text { generalizations. Among such generalizations is the best-known BLYM inequality and } \\
\text { the Ahlswede--Zhang (AZ) identity which surprisingly generalizes the BLYM into an } \\
\text { identity. Sperner's theorem and the BLYM inequality has been also generalized to a } \\
\text { wide class of posets. Another direction in this research was the study of more part } \\
\text { Sperner systems. In this paper we derive AZ type identities for regular posets. We also } \\
\text { characterize all maximum 2-part Sperner systems for a wide class of product posets. }\end{array}$ \\
\hline
\end{tabular}


1

2

3

\title{
AZ-identities and Strict 2-part Sperner Properties of Product Posets ${ }^{\text {th }}$
}

\author{
Harout Aydinian ${ }^{\mathrm{a}, 1}$, Péter L. Erdős ${ }^{\mathrm{b}, 2}$ \\ ${ }^{a}$ Department of Mathematics, University of Bielefeld, POB 100131, D-33501, Bielefeld \\ email: ayd@math.uni-bielefeld.de \\ ${ }^{b}$ Alfréd Rényi Institute, Reáltanoda u 13-15 Budapest, 1053 Hungary \\ email: elp@renyi.hu
}

\begin{abstract}
One of central issues in extremal set theory is Sperner's theorem and its generalizations. Among such generalizations is the best-known BLYM inequality and the Ahlswede-Zhang (AZ) identity which surprisingly generalizes the BLYM into an identity. Sperner's theorem and the BLYM inequality has been also generalized to a wide class of posets. Another direction in this research was the study of more part Sperner systems. In this paper we derive AZ type identities for regular posets. We also characterize all maximum 2-part Sperner systems for a wide class of product posets.
\end{abstract}

Key words: Sperner property; strict Sperner property; strict BLYM inequality; AZ-identity; 2-part Sperner property; normal poset; regular poset

\section{Introduction}

Extremal set theory began in 1928 with Sperner's seminal result in [23]. From that time dozens of generalizations were discovered (a comprehensive survey of these results can be found in excellent book [8].) The best-known generalization of Sperner's theorem is the BLYM inequality due to Bollobas [5], Lubell [19], Meshalkin [20], and Yamamoto [24]. An elegant result discovered by Ahlswede and Zhang $([1,2])$ constitutes equalities for any subset system, which, in turn, directly infers not only the corresponding BLYM

\footnotetext{
${ }^{\text {th }}$ Part of these results was published in an extended abstract form in the Proc. of Eurocomb 2011.

${ }^{1}$ This author was supported by DFG-project AH46/7-1 "General Theory of Information Transfer"

${ }^{2}$ During this research PLE enjoyed the hospitality of the AG Genominformatik of Universität Bielefeld, Germany and was supported in part by the Alexander von Humboldt Foundation and by the Hungarian NSF, under contract NK 78439 and K 68262.
} 
inequalities, but also the strict versions. Sperner property and related problems for posets was intensively studied by many authors. Another direction in this research was the study of more part Sperner systems, which was motivated by an applications of Sperner type result in 1945 by Erdős [9].

In Section 2 we derive AZ type identities for regular posets. We also discuss strict Sperner properties and strict BLYM inequalities for a subclass of normal posets. In Section 3 we present a 2-part AZ-identity for regular posets. Finally, we characterize all maximum size 2-part Sperner systems for products of two strictly normal posets.

\section{Sperner systems and related problems in posets}

Let $(\mathcal{P}, \leq)$ be a poset with a rank function $r$. We denote the set of elements of rank $i$ by $\mathcal{P}_{i} ; i=0,1, \ldots, r(\mathcal{P})$ where $r(\mathcal{P})$ is the maximum rank in $\mathcal{P}$. Also $N_{i}(\mathcal{P})$ will denote the $i$ th Whitney number of $\mathcal{P}$, that is $N_{i}(\mathcal{P})=\left|\mathcal{P}_{i}\right|$, and for short we will use $N_{i}$ when this does not cause confusion. In the sequel sometimes we associate $\mathcal{P}$ with its Hasse diagram and use the corresponding terminology. In particular the Hasse diagram can be represented as a series of bipartite graphs $G_{i, i+1}(\mathcal{P})=\left(\mathcal{P}_{i}, \mathcal{P}_{i+1} ; E(\prec)\right)$ (for $i=0, \ldots, r(\mathcal{P})-1$ ) where $\prec$ denotes the cover relation between the consecutive levels, that is $(a, b) \in E(\prec)$ iff $a \prec b$ (in words: if $b$ covers $a$ ).

For the lower (resp. upper) degree of an element $a \in \mathcal{P}$ we use the notation $d^{-}(a)$ (resp. $d^{+}(a)$ ). For an element $a \in \mathcal{P}$ we define $\Gamma_{i}^{+}(a)=$ $\left\{x \in \mathcal{P}_{i}: x \geq a\right\}$ and $\Gamma_{i}^{-}(a)=\left\{x \in \mathcal{P}_{i}: x \leq a\right\}$. Similarly, for a subset $A \subset \mathcal{P}$ we define $\Gamma_{i}^{+}(A)=\left\{x \in \mathcal{P}_{i}: x \geq a\right.$ for some $\left.a \in A\right\}$ and $\Gamma_{i}^{-}(A)=$ $\left\{x \in \mathcal{P}_{i}: x \leq a\right.$ for some $\left.a \in A\right\}$. We also put $\Gamma^{+}(a)=\Gamma_{r(a)+1}^{+}(a)$ and $\Gamma^{-}(a)=\Gamma_{r(a)-1}^{-}(a)$. In fact, $d^{+}(a)=\left|\Gamma^{+}(a)\right|$ and $d^{-}(a)=\left|\Gamma^{-}(a)\right|$. Given a subset $A \subset \mathcal{P}$ we denote by $\mathcal{U}(A)$ the upset (also called filter) generated by $A$, that is $\mathcal{U}(A)=\{b \in \mathcal{P}: b \geq a, a \in A\}$. Similarly is defined the downset (also called ideal) $\mathcal{D}(A)=\{b \in \mathcal{P}: b \leq a, a \in A\}$.

A poset $\mathcal{P}$ is called regular if for every element $a \in \mathcal{P}$ of a given rank, both the lower and upper degrees of $a$ depend on its rank $r(a)$, but not on the actual choice of $a$ within the level. For a regular poset $\mathcal{P}$ we use $d_{i}^{-}$ (resp. $d_{i}^{+}$) for the lower degree (resp. upper degree) of an element in $\mathcal{P}_{i}$.

A poset $\mathcal{P}$ is called normal (introduced in [12]) if it satisfies the normalized matching property, that is for every $A \subset \mathcal{P}_{i}$ we have $|A| / N_{i} \leq$ $\left|\Gamma_{i-1}^{-}(A)\right| / N_{i-1}$. The definition implies that every normal poset $\mathcal{P}$ is graded, that is all minimal and maximal elements have rank 0 and $r(\mathcal{P})$, respectively. Thus, each maximal chain in $\mathcal{P}$ has length $r(\mathcal{P})+1$. It is easy to see that each regular poset $\mathcal{P}$ is normal.

A subset $C \subset \mathcal{P}$ with $|C|=k$ is called a chain of length $k$ if its members are pairwise comparable. A chain is called maximal if it is not contained in a larger chain. A subset $A \subset \mathcal{P}$ is called an antichain or a Sperner system if its 
members are pairwise incomparable. A subset $A \subset \mathcal{P}$ is called a $k$-Sperner system if it contains no chain of length $k+1$. A subset $A \subset \mathcal{P}$ is called homogeneous if it consists of the union of complete levels. Clearly if $A$ is a homogeneous system of $k$ levels then it is a $k$-Sperner system. A poset is said to have strong Sperner property if for every $k$ there exists a maximum size homogeneous $k$-Sperner system. It is said to have strict $k$-Sperner property if all maximum $k$-Sperner systems are homogeneous.

\subsection{AZ-identities for regular posets}

In this subsection we prove two Ahlswede-Zhang type equalities for regular posets. At first we need the following notation. Given $A \subset \mathcal{P}$ and $x \in \mathcal{P}$, we denote $A^{x}=\{a \in A: a \leq x\}$. We also define $W_{A}(x)=$ $\left|\Gamma^{-}(x) \backslash \Gamma_{r(x)-1}^{+}\left(A^{x}\right)\right|$.

Theorem 2.1. Let $\mathcal{P}$ be a regular poset and let $A \subset \mathcal{P}$. Then we have

$$
\sum_{x \in \mathcal{P}} \frac{W_{A}(x)}{d_{r(x)}^{-} N_{r(x)}}=1 .
$$

By convention, for $x \in \mathcal{P}_{0}$ (thus $\left.W_{A}(x)=0, d_{r(x)}^{-}=0\right)$ we put $\frac{W_{A}(x)}{d_{0}^{-} N_{0}}:=\frac{1}{N_{0}}$.

It is not hard to see that when our poset is the Boolean lattice $2^{[n]}$, then

$$
W_{A}(x)=\left|\bigcap_{x \supseteq a \in A} a\right|, d_{r(x)}^{-}=|x|, N_{r(x)}=\left(\begin{array}{c}
n \\
|x|
\end{array}\right)
$$

and (2.1) reduces to the original Ahlswede-Zhang identity (see [1]). Notice also that if $A$ is an antichain then $W_{A}(x)=d_{r(x)}^{-}$for all $x \in A$ and hence we have the following identity for antichains.

\section{Corollary 2.2.}

$$
\sum_{x \in A} \frac{1}{N_{r(x)}}+\sum_{x \in \mathcal{P} \backslash A} \frac{W_{A}(x)}{d_{r(x)}^{-} N_{r(x)}}=1 .
$$

This in particular implies the LYM inequality for regular posets:

$$
\sum_{x \in A} \frac{1}{N_{r(x)}} \leq 1
$$

which seemingly first appeared in Baker [4].

Remark 2.3. It is important to recognize that Theorem 2.1 does not hold for all normal posets. For example the poset on Figure 1 (a) is normal, however does not have this property. Indeed, take the antichain $A=\{a, c\}$. 
Then $W_{A}(a)=W_{A}(b)=W_{A}(c)=1$ and $N_{r(a)}=N_{r(b)}=N_{r(c)}=2$, furthermore, $d_{r(a)}^{-}=d_{r(c)}^{-}=1$ and $d_{r(b)}^{-}=2$. Therefore,

$$
\sum_{x \in P} \frac{W_{A}(x)}{d_{r(x)}^{-} N_{r(x)}}=1 / 2+1 / 2+1 / 4>1 .
$$

Proof of Theorem 2.1. We follow directly the idea of the proof in [1]. Let $\mathcal{P}$ be a regular lattice and let $n:=r(\mathcal{P})$. Denote by $\mathcal{E}_{A}$ the boundary edges of $\mathcal{U}(A)$, that is the edges between $\mathcal{U}(A)$ and $\mathcal{P} \backslash \mathcal{U}(A)$. Furthermore, for any given $u \in \mathcal{U}(A)$ let $\mathcal{E}_{A}(u)=\left\{(u, v) \in \mathcal{E}_{A}\right\}$.

Note now that the number of maximal chains passing through the boundary edges $\mathcal{E}_{A}$ is equal to the number of all maximal chains. Observe also that for each $x \in \mathcal{U}(A)$ the number of maximal chains passing through the edges $\mathcal{E}_{A}(x)$ is $d_{r(x)}^{+} \ldots d_{n-1}^{+} \cdot\left|\mathcal{E}_{A}(x)\right| \cdot d_{r(x)-1}^{-} \ldots d_{1}^{-}$. The number of maximal chains in a regular poset $\mathcal{P}$ is $N_{0} d_{0}^{+} d_{1}^{+} \ldots d_{r(\mathcal{P})-1}^{+}=N_{r(\mathcal{P})} d_{r(\mathcal{P})}^{-} d_{r(\mathcal{P})-1}^{-} \ldots d_{1}^{-}$. Hence we have

$$
\sum_{x \in \mathcal{U}(A)} d_{r(x)}^{+} \ldots d_{n-1}^{+} \cdot\left|\mathcal{E}_{A}(x)\right| \cdot d_{r(x)-1}^{-} \ldots d_{1}^{-}=N_{0} d_{0}^{+} d_{1}^{+} \ldots d_{n-1}^{+} .
$$

Since $\mathcal{E}_{A}(x)=\emptyset$ for $x \notin \mathcal{U}(A)$, we can rewrite this as

$$
\sum_{x \in \mathcal{P}} \frac{\left|\mathcal{E}_{A}(x)\right| d_{1}^{-} \ldots d_{r(x)-1}^{-}}{N_{0} d_{0}^{+} d_{1}^{+} \ldots d_{r(x)-1}^{+}}=1 .
$$

Observe that, in view of regularity, for every $1 \leq k \leq n$ the following holds

$$
N_{k}=\frac{N_{0} d_{0}^{+} \ldots d_{k-1}^{+}}{d_{1}^{-} \ldots d_{k}^{-}} .
$$

Therefore, (2.4) gives

$$
\sum_{x \in \mathcal{P}} \frac{\left|\mathcal{E}_{A}(x)\right|}{d_{r(x)}^{-} N_{r(x)}}=1 .
$$

To finish the proof it remains to show that $\left|\mathcal{E}_{A}(x)\right|=W_{A}(x)$. The latter is clear since $\Gamma_{r(x)-1}^{+}\left(A^{x}\right) \subset \mathcal{U}(A)$ and hence $\mathcal{E}_{A}(x)=\Gamma^{-}(x) \backslash \Gamma_{r(x)-1}^{+}\left(A^{x}\right)$.

The reader can easily observe that using the same approach we can extend Theorem 2.1 (as well as Corollary 2.2) to a larger class of posets as follows.

Theorem 2.4. Let $\mathcal{P}$ be a poset such that each element of rank in $\mathcal{P}_{i}$ is contained in the same number of maximal chains for all $i$. Then for a nonempty subset $A \subset \mathcal{P}$ we have

$$
\sum_{x \in \mathcal{P}} \frac{W_{A}(x)}{d^{-}(x) N_{r(x)}}=1 .
$$


Note next that equality (2.2) can be extended to $k$-Sperner systems. By the dual version of the Dilworth theorem (attributed to Erdős and Szekeres see [18] Ex. 9.32b), we can decompose such a system $A$ into $k$ disjoint antichains $A=A_{1} \cup \ldots \cup A_{k}$. Applying (2.2) to each antichain $A_{i}$ and then summing up all $k$ identities we get the following identity which in turn implies $k$-BLYM inequality for regular posets.

Corollary 2.5. Let $A$ be $k$-Sperner system in a regular poset $\mathcal{P}$. Then we have

$$
\sum_{x \in A} \frac{1}{N_{r(x)}}+\sum_{i=1}^{k} \sum_{x \in \mathcal{P} \backslash A_{i}} \frac{W_{A}(x)}{d_{r(x)}^{-} N_{r(x)}}=k
$$

Ahlswede and Zhang found yet another generalization of identity (2.1) for the Boolean lattice (see [2]) which in turn is a sharpening of the Bollobás inequality [5]. This latter result is based on a higher level regularity of the Boolean lattice, therefore we also have to restrict ourselves to a subclass of regular posets with stronger regularities. Also we consider now lattices.

We call a lattice $\mathcal{P}$ strongly regular if for all pairs $a, b \in \mathcal{P}$, with $a<b$, the number $\left|\Gamma_{i}^{+}(a) \cap \Gamma_{j}^{-}(b)\right|$ depends only on $i, j$ and $r(a), r(b)$.

Thus, we can define the quantity $\lambda_{i}(k, l)$ for all integers $0 \leq k \leq l \leq r(\mathcal{P})$ and $k \leq i \leq l$ as $\lambda_{i}(k, l)=\left|\Gamma_{i}^{+}(a) \cap \Gamma_{i}^{-}(b)\right|$, where $a, b \in \mathcal{P}, a \leq b$ and $r(a)=k, r(b)=l$ (by convention we put here $\Gamma_{r(x)}^{+}(x)=\Gamma_{r(x)}^{-}(x)=x$ for all $x \in \mathcal{P})$. For all other triples $(i, k, l)$ of integers we put $\lambda_{i}(k, l)=0$. Note that a strongly regular lattice is regular.

Theorem 2.6. Let $A=\left\{a_{1}, \ldots, a_{m}\right\}$ and $B=\left\{b_{1}, \ldots, b_{m}\right\}$ be subsets of $a$ strongly regular lattice $\mathcal{P}$ such that $a_{i} \leq b_{j}$ iff $i=j$. Let also $k_{i}:=r\left(a_{i}\right)$ and $l_{i}:=r\left(b_{i}\right) ; i=1, \ldots, m$. Then we have

$$
\sum_{i=1}^{m} \beta\left(k_{i}, l_{i}\right)+\sum_{x \in \mathcal{U}(A) \backslash \mathcal{D}(B)} \frac{W_{A}(x)}{d_{r(x)}^{-} N_{r(x)}}=1,
$$

where

$$
\beta\left(k_{i}, l_{i}\right):=\sum_{j=0}^{l_{i}-k_{i}} \frac{\lambda_{k_{i}+j}\left(k_{i}, l_{i}\right)}{\left(k_{i}+j\right) N_{k_{i}+j}} \cdot\left(d_{r(x)}^{-}-\lambda_{k_{i}+j-1}\left(k_{i}, k_{i}+j\right)\right) .
$$

Proof. For every pair $a, b \in \mathcal{P}$ with $a \leq b$ let us denote $S(a, b)=\{x \in \mathcal{P}$ : $a \leq x \leq b\}$. By the definition of strong regularity

$$
|S(a, b)|=\sum_{j=0}^{r(b)-r(a)} \lambda_{r(a)+j}(r(a), r(b))
$$


Note also that (by the condition of the theorem) $S\left(a_{i}, b_{i}\right) \cap S\left(a_{j}, b_{j}\right)=\emptyset$ for all $i \neq j(i, j \in[m])$. We proceed now like in the proof of Theorem 2.1 counting all maximal chains.

We count first all maximal chains that meet $S\left(a_{i}, b_{i}\right)$ for $i=1, \ldots, m$. This number (by the same arguments as in the proof of Theorem 2.1) equals

$$
\sum_{a_{i} \leq x \leq b_{i}} \frac{W_{A}(x)}{d_{r(x)}^{-} N_{r(x)}} \cdot d_{0}^{+} d_{1}^{+} \ldots d_{n-1}^{+} .
$$

Note that these $m$ sets of maximal chains are pairwise disjoint. The number of remaining maximal chains equals

$$
\sum_{x \in \mathcal{U}(A) \backslash \mathcal{D}(B)} \frac{W_{A}(x)}{d_{r(x)}^{-} N_{r(x)}} \cdot d_{0}^{+} d_{1}^{+} \ldots d_{n-1}^{+} .
$$

Thus, we have

$$
\sum_{i=1}^{m} \sum_{a_{i} \leq x \leq b_{i}} \frac{W_{A}(x)}{d_{r(x)}^{-} N_{r(x)}}+\sum_{x \in \mathcal{U}(A) \backslash \mathcal{D}(B)} \frac{W_{A}(x)}{d_{r(x)}^{-} N_{r(x)}}=1 .
$$

Observe now that for each $a_{i} \leq x \leq b_{i}$ we have $W_{A}(x)=d_{r(x)}^{-}-\lambda_{r(x)-1}\left(k_{i}, r(x)\right)$. Hence, we get

$$
\sum_{a_{i} \leq x \leq b_{i}} \frac{W_{A}(x)}{d_{r(x)}^{-} N_{r(x)}}=\beta\left(k_{i}, l_{i}\right) ; i=1, \ldots, m .
$$

This completes the proof.

Again, when we apply this theorem to the Boolean lattice, we get back verbatim the original second AZ-identity (see [2]), since in this case we have

$$
\begin{aligned}
& \beta\left(k_{i}, l_{i}\right)=\sum_{a_{i} \subseteq x \subseteq b_{i}} \frac{\left|a_{i}\right|}{|x|\left(\begin{array}{c}
n \\
|x|
\end{array}\right)}= \\
& =\sum_{k=0}^{\left|b_{i}\right|-\left|a_{i}\right|}\left(\begin{array}{c}
\left|b_{i}\right|-\left|a_{i}\right| \\
k
\end{array}\right) \cdot \frac{\left|a_{i}\right|}{\left(\left|a_{i}\right|+k\right)\left(\begin{array}{c}
n \\
\left|a_{i}\right|+k
\end{array}\right)}=\frac{1}{\left(\begin{array}{c}
n-\left|b_{i}\right|+\left|a_{i}\right| \\
\left|a_{i}\right|
\end{array}\right)} .
\end{aligned}
$$

\subsection{Strict BLYM inequalities for normal posets}

In this subsection we turn our attention to strict Sperner theorems and strict BLYM inequalities. We start with a strong result of Kleitman (which we use later), which in fact extends the BLYM inequality to a wide class of posets. The following notion was introduced in Kleitman [17]. 
Definition 2.7. If $\mathfrak{C}(\mathcal{P})$ denotes the set of maximal chains in a graded poset $\mathcal{P}$ then a regular covering of $P$ is a function $f: \mathfrak{C}(P) \rightarrow \mathbb{R}_{+} \cup\{0\}$ such that the following holds:

$$
\text { (a) } \sum_{C \in \mathfrak{C}} f(C)=1 ; \quad(b) \forall a \in P: \sum_{C \in \mathfrak{C}: a \in C} f(C)=\frac{1}{N_{r(a)}} .
$$

Theorem 2.8 (Kleitman, [17]). A poset satisfies the $k$-BLYM inequalities for all possible $k$ if and only if it is a normal poset, which, in turn, is equivalent to the existence of a regular chain covering.

Recall that any regular poset is normal, so Kleitman's theorem applies for them. It is important to recognize that normality does not always imply that the poset also satisfies the strict BLYM inequality, or strict Sperner property. In fact, none of the normal posets on Figure 1 satisfy the strict Sperner property.

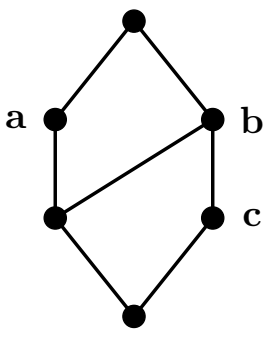

(a)

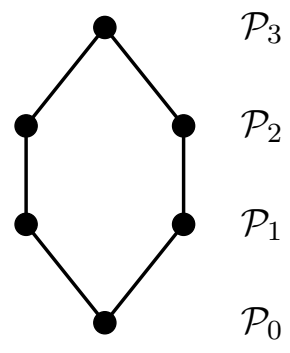

(b)

Figure 1: These posets are not strict Sperner. (a) normal, but not regular poset, the first AZ-identity does not hold; (b) normal, but not levelconnected poset, since the induced bipartite graph $G_{1,2}$ is not connected. Neither poset satisfies the strict Sperner property.

The study of the strict Sperner property of normal posets was initiated by Engel $([6,7])$. He introduced the following strengthening of the normalized matching property:

Definition 2.9. A normal poset $\mathcal{P}$ is called strictly normal if it satisfies the strict normalized matching property, that is for every proper subset $A \subset \mathcal{P}_{i} ; i \in\{1, \ldots, r(\mathcal{P})\}$ we have $|A| / N_{i}<\left|\Gamma^{-}(A)\right| / N_{i-1}$.

Theorem 2.10 (Engel [6]). Every strictly normal poset has strict $k$-Sperner property.

Furthermore Engel also proved ([7], [8, Ch. 4.6]) that the direct product of strictly normal posets with log-concave Whitney numbers is again a strictly normal posets with log-concave Whitney numbers. Therefore, Theorem 2.10 holds for the direct products of such posets.

The following easy result will be used in Section 3. 
Theorem 2.11. Let $\mathcal{P}$ be a strictly normal poset. Then $\mathcal{P}$ satisfies the strict $k$-LYM inequality, that is if $A \subset \mathcal{P}$ is a $k$-Sperner system such that

$$
\sum_{a \in F} \frac{1}{N_{r(a)}}=k
$$

holds, then $A$ is homogeneous.

Proof. By the dual Dilworth theorem, $F$ can be decomposed into $k$ pairwise disjoint antichains. Then equality (2.8) can be written as the sum of $k$ BLYM inequalities, which in fact are equalities. Let $A$ be one of antichains in the decomposition of $F$, thus $\sum_{a \in A} \frac{1}{N_{r(a)}}=1$. Suppose now that $A$ contains elements from different levels and let $B \subset \mathcal{P}_{i}$ be the elements of the highest level present in $A$. Replacing $B$ by $\Gamma^{-}(B)$ in $A$ we get a new Sperner system $A^{\prime}$. However, in view of strict normality, we have now $\sum_{a \in A^{\prime}} \frac{1}{N_{r(a)}}>1$, a contradiction with Theorem 2.8 .

The strict normalized matching property seems to be a rather strong prerequisite. How can one find such posets? The following notion (introduced in $[6],[7]$ ) leads to a subclass of regular posets that satisfy this property.

Definition 2.12. A poset $\mathcal{P}$ is called level-connected if for each $i$ the bipartite graph $G_{i, i+1}(\mathcal{P})$ is connected.

The following fact is a special case of a more general statement in [6].

Lemma 2.13. A poset is strictly normal if it is regular and level-connected.

Thus, we have the following:

Corollary 2.14. Let $\mathcal{P}$ be a regular level-connected poset. Then it satisfies the strict $k-L Y M$ inequality.

Remark 2.15. Using the same approach as in Lemma 3.6 (in Subsection 3.2 ), we can also show that for a maximum size $k$-Sperner system $F$ in a normal poset the equality $\sum_{a \in F} \frac{1}{N_{r(a)}}=k$ holds. Applying now Theorem 2.11 for a strictly normal poset we get Theorem 2.10 .

\section{Two-part problems}

Let $S=S_{1} \uplus S_{2}$ be a fixed partition of the underlying $n$-element set $S$. A family $\mathcal{H}$ of subsets of $S$ is called a 2-part Sperner family if

$$
\forall E, F \in \mathcal{H} \quad\left(E \varsubsetneqq F \Rightarrow \forall i: F \backslash E \nsubseteq S_{i}\right) .
$$

Katona [14] and Kleitman [16] proved independently around 1965 that no 2-part Sperner system in the Boolean lattice can be bigger than a maximum size Sperner family. 
Much later, only in 1986, proved P.L. Erdös and Katona in [11] that the 2-part Sperner theorem in the Boolean lattice is strict, that is all optimal $2-$ part Sperner families are homogeneous. Here, homogeneous means that the family is a union of products of complete levels in $2^{S_{1}}$ and $2^{S_{2}}$.

In 1971 Schonheim proved (see [22]) the 2-part Sperner theorem for the direct product of two divisor lattices: here a subset $A \subset \mathcal{P}_{1} \times \mathcal{P}_{2}$ is called ${ }^{2}$ part Sperner system if whenever both components of $(x, y) \in A$ are smaller than the corresponding components of $(u, v) \in A$ then nor $x=u$ neither $y=v$ hold. Katona in [15] generalized this result for the direct product of symmetric chain posets.

\subsection{2-part AZ-identity}

In this subsection we derive a 2-part AZ type identity for direct products of regular posets.

Let $\mathcal{P}$ and $\mathcal{Q}$ be regular posets and let $A \subset \mathcal{P} \times \mathcal{Q}$. For each $y \in \mathcal{Q}$ we define $A(y)=\{a \in \mathcal{P}:(a, y) \in A\}$ and $A^{x}(y)=\{a \in \mathcal{P}:(a, y) \in A, a \leq x\}$. Furthermore, for a given $(x, y) \in \mathcal{P} \times \mathcal{Q}$ we define $W_{A(y)}(x)=\left|\Gamma^{-}(x)\right|$ $\Gamma_{r(x)-1}^{+}\left(A^{x}(y)\right) \mid$.

Theorem 3.1. For regular posets $\mathcal{P}$ and $\mathcal{Q}$ and a subset $A \subset \mathcal{P} \times \mathcal{Q}$ we have

$$
\sum_{(x, y) \in \mathcal{P} \times \mathcal{Q}} \frac{W_{A(y)}(x)}{d_{r(x)}^{-} N_{r(x)}^{(1)} N_{r(y)}^{(2)}}=r(\mathcal{Q})+1 .
$$

By convention, for $x \in \mathcal{P}_{0}$ we put $\frac{W_{A}(x)}{d_{0}^{-} N_{0}^{(1)} N_{r(y)}^{(2)}}:=\frac{1}{N_{0}^{(1)} N_{r(y)}^{(2)}}$.

Proof. For any given $y \in \mathcal{Q}_{i}$ we can apply the AZ-identity (2.1) for the subset $A(y) \subset \mathcal{P}$. Summing up the corresponding identities for all $y \in \mathcal{Q}_{i}$ we get:

$$
\sum_{(x, y) \subset \mathcal{P} \times \mathcal{Q}: y \in \mathcal{Q}_{i}} \frac{W_{\mathcal{A}(y)}(x)}{d_{r(x)}^{-} N_{r(x)}^{(1)} N_{r(y)}^{(2)}}=1 .
$$

We do the same for all levels $i=0,1, \ldots, r(\mathcal{Q})$ in $\mathcal{Q}$ and then sum up all these identities. This gives the result.

It it easy to see that the latter implies the following identity for 2-part Sperner systems in a product of two regular posets.

Corollary 3.2. Let $\mathcal{P}$ and $\mathcal{Q}$ be regular posets. Let also $N_{i}^{(1)}:=\left|\mathcal{P}_{i}\right|$, $N_{i}^{(2)}:=\left|\mathcal{Q}_{i}\right|$, and $r(\mathcal{Q}) \leq r(\mathcal{P})$. If $A \subset \mathcal{P} \times \mathcal{Q}$ is a 2-part Sperner system, then

$$
\sum_{(a, b) \in A} \frac{1}{N_{r(a)}^{(1)} N_{r(b)}^{(2)}}+\sum_{(x, y) \notin A} \frac{W_{A(y)}(x)}{d_{r(x)}^{-} N_{r(x)}^{(1)} N_{r(y)}^{(2)}}=r(\mathcal{Q})+1 .
$$




\subsection{A strict 2-part Sperner theorem}

In this subsection we generalize the strict 2-part Sperner theorem (see [11]) for direct products of two strictly normal posets. The proof follows closely the proof of the original strict 2-part Sperner theorem in [3].

Theorem 3.3. Let $\mathcal{P}$ and $\mathcal{Q}$ be strictly normal posets and let $F \subset \mathcal{P} \times \mathcal{Q}$ be a maximum size 2-part Sperner system. Then $F$ is a homogeneous system.

In fact, the theorem allows us to characterize all maximum size 2-part Sperner systems in the described subclasses of normal posets. To do this we define below the notion of the well-paired homogeneous system.

Let $F=\bigcup_{(i, j) \in I} \mathcal{P}_{i} \times Q_{j}$. Then clearly $F$ is a 2 -part Sperner system if and only if $I$ is a transversal, that is every two members of $I$ do not have a component in common. Clearly $|I| \leq \min \{r(\mathcal{P}), r(\mathcal{Q})\}+1$ and in the case of equality, $I$ is called a full transversal (otherwise it is called partial). Note that if $F$ is a maximum size homogeneous system, then $I$ is a full transversal, since every partial transversal can be extended to a full one. Now clearly $|F|=\max _{I} \sum_{(i, j) \in I}\left|P_{i}\right|\left|Q_{j}\right|$ where $I \subset\{0, \ldots, r(\mathcal{P})\} \times\{0, \ldots, r(\mathcal{Q})\}$ is a full transversal. Thus, an optimal $F$ consists of union of products of full levels in each poset, such that $|I|$ largest levels in $\mathcal{P}$ are paired with corresponding $|I|$ largest levels in $\mathcal{Q}$. We call such a 2 -part Sperner system well-paired. In fact, we have seen that the following holds.

Lemma 3.4. Let $\mathcal{P}, \mathcal{Q}$ be ranked posets and let $F \subset \mathcal{P} \times \mathcal{Q}$ be a maximum size homogeneous 2-part Sperner system. Then $F$ is a well paired system.

We can rephrase now Theorem 3.3 as follows:

(†) All maximum size 2-part Sperner systems in the described classes of product posets are well-paired .

We prove Theorem 3.3 through a series of lemmas. Let $\mathfrak{C}:=\mathfrak{C}_{1} \times \mathfrak{C}_{2}$ where $\mathfrak{C}_{1}:=\mathfrak{C}(\mathcal{P}), \mathfrak{C}_{2}:=\mathfrak{C}(\mathcal{Q})$ and let $n_{1}:=r(\mathcal{P}), n_{2}:=r(\mathcal{Q})$.

Lemma 3.5. Let $\mathcal{P}, \mathcal{Q}$ be normal posets and let $F \subset \mathcal{P} \times \mathcal{Q}$ be a 2-part Sperner system. Let also $\left(C_{1}, C_{2}\right) \in \mathfrak{C}(\mathcal{P}) \times \mathfrak{C}(\mathcal{Q})$. If $A:=F \cap\left(C_{1} \times C_{2}\right)$ then

$$
|A| \leq \min \{r(\mathcal{P}), r(\mathcal{Q})\}+1
$$

Proof. Let $C_{1}$ and $C_{2}$ consist of elements $a_{0}<a_{1}<\ldots<a_{n_{1}}$ and $b_{0}<b_{1}<$ $\ldots<b_{n_{2}}$ respectively. Let $I=\left\{(i, j) \in\left(n_{1}+1\right) \times\left(n_{2}+1\right):\left(a_{i}, b_{j}\right) \in A\right\}$. Observe now that this $I$ is a transversal. Hence the result.

The following result plays a key role in our proof. 
Lemma 3.6. Let $\mathcal{P}, \mathcal{Q}$ be normal posets and let $F \subset \mathcal{P} \times \mathcal{Q}$ be a maximum size 2-part Sperner system. Assume also that $n_{2} \leq n_{1}$. Then

$$
\sum_{(a, b) \in F} \frac{1}{N_{r(a)}^{(1)} N_{r(b)}^{(2)}}=n_{2}+1 .
$$

Remark 3.7. To prove this equality for Boolean lattices in [3] was used results from the theory of convex hulls for 2-part Sperner families ([10]). Here we could apply similar arguments, using in addition a result of Sali $([21])$. For the sake of completeness, we give her a direct proof of this statement.

Proof. Let $f_{1}, f_{2}$ be regular chain coverings of $\mathcal{P}$ and $\mathcal{Q}$ respectively. Define $g\left(C_{1}, C_{2}\right)=f_{1}\left(C_{1}\right) f_{2}\left(C_{2}\right)$ for all $\left(C_{1}, C_{2}\right) \in \mathfrak{C}_{1} \times \mathfrak{C}_{2}$. Let $F \subset \mathcal{P} \times \mathcal{Q}$ be a maximum size 2 -part Sperner system. Then in view of regular chain coverings $f_{1}$ and $f_{2}$ we have

$$
\begin{aligned}
& |F|=\sum_{(a, b) \in F} 1=\sum_{(a, b) \in F}\left(\sum_{\substack{C_{1} \in \mathfrak{c}_{1}: \\
a \in C_{1}:}} f_{1}\left(C_{1}\right) N_{r(a)}^{(1)}\right)\left(\sum_{\substack{C_{2} \in \mathfrak{C}_{2}: \\
a \in C_{2}}} f_{2}\left(C_{2}\right) N_{r(a)}^{(2)}\right) \\
& =\sum_{(a, b) \in F}\left(\sum_{\substack{\left(C_{1}, C_{2}\right) \in \mathfrak{C}: \\
(a, b) \in\left(C_{1} \times C_{2}\right)}} g\left(C_{1}, C_{2}\right) N_{r(a)}^{(1)} N_{r(b)}^{(2)}\right) \\
& =\sum_{\left(C_{1}, C_{2}\right) \in \mathfrak{C}}\left(g\left(C_{1}, C_{2}\right) \sum_{(a, b) \in\left(C_{1} \times C_{2}\right) \cap F} N_{r(a)}^{(1)} N_{r(b)}^{(2)}\right) \\
& \leq \sum_{\left(C_{1} \times C_{2}\right) \cap F \neq \emptyset} g\left(C_{1}, C_{2}\right)\left(\max _{\left(C_{1}, C_{2}\right) \in \mathfrak{C}} \sum_{(a, b) \in\left(C_{1} \times C_{2}\right) \cap F} N_{r(a)}^{(1)} N_{r(b)}^{(2)}\right) \\
& \leq \max _{\left(C_{1}, C_{2}\right) \in \mathfrak{C}} \sum_{(a, b) \in\left(C_{1} \times C_{2}\right) \cap F} N_{r(a)}^{(1)} N_{r(b)}^{(2)} \text {. }
\end{aligned}
$$

Before we continue the proof of Lemma 3.6 we notice that the last inequality together with Lemma 3.4 implies the following:

Corollary 3.8. In the product of two normal posets there exist maximum size 2-part Sperner systems which are well-paired (homogeneous) systems.

Now, since $F$ has maximum size we must have everywhere equalities. From this we infer an important consequence:

Lemma 3.9. For any maximum size 2-part Sperner system $F \subset \mathcal{P} \times \mathcal{Q}$ we have $\left|\left(C_{1} \times C_{2}\right) \cap F\right|=n_{2}+1$ for all $\left(C_{1}, C_{2}\right) \in \mathfrak{C}_{1} \times \mathfrak{C}_{2}$ with $g\left(C_{1}, C_{2}\right)>0$. 
Proof. Indeed, the equality in (3.4) implies that for each pair $\left(C_{1}, C_{2}\right)$ with $\left(C_{1} \times C_{2}\right) \cap F \neq \emptyset$ we have

$$
\sum_{(a, b) \in\left(C_{1} \times C_{2}\right) \cap F} N_{r(a)}^{(1)} N_{r(b)}^{(2)}=\left|F^{*}\right|,
$$

where $F^{*}$ is an optimal homogeneous 2-part Sperner system. Since, in view of Lemma 3.4, $F^{*}$ is full, it implies that $\left|\left\{(a, b) \in\left(C_{1} \times C_{2}\right)\right\}\right|=n_{2}+1$. On the other hand, an equality in (3.5) implies that

$$
\sum_{\left(C_{1} \times C_{2}\right) \cap F \neq \emptyset} g\left(C_{1}, C_{2}\right)=1,
$$

which means that $\left(C_{1} \times C_{2}\right) \cap F \neq \emptyset$ for each pair $\left(C_{1}, C_{2}\right)$ with $g\left(C_{1}, C_{2}\right)>0$. This completes the proof of Lemma 3.9.

Now we are going to finish the proof of Lemma 3.6. We have

$$
\begin{aligned}
\sum_{(a, b) \in F} \frac{1}{N_{r(a)}^{(1)} N_{r(b)}^{(2)}} & \left.=\sum_{(a, b) \in F}\left(\sum_{\substack{\left(C_{1}, C_{2}\right) \in \mathfrak{C}: \\
(a, b) \in\left(C_{1} \times C_{2}\right)}} g\left(C_{1}, C_{2}\right) N_{r(a)}^{(1)} N_{r(b)}^{(2)}\right)\right) \frac{1}{N_{r(a)}^{(1)} N_{r(b)}^{(2)}} \\
& =\sum_{(a, b) \in F} \sum_{\substack{\left(C_{1}, C_{2}\right) \in \mathfrak{C}: \\
(a, b) \in\left(C_{1} \times C_{2}\right)}} g\left(C_{1}, C_{2}\right) \\
& =\sum_{\left(C_{1}, C_{2}\right) \in \mathfrak{C}} g\left(C_{1}, C_{2}\right) \\
& =\sum_{\left(n_{2}+1\right)}\left(\sum_{(a, b) \in\left(C_{1} \times C_{2}\right) \cap F} 1\right) \\
\left(C_{1} \times C_{2}\right) \cap F \neq \emptyset & \left.g\left(C_{1}, C_{2}\right)\right)=n_{2}+1
\end{aligned}
$$

Here in the first line we applied the Definition 2.7(b). The second sum in equality (3.7) is $n_{2}+1$, due to Lemma 3.9 , finally the sum in equality (3.8) is 1 because of equality (3.6). We are done with Lemma 3.6.

Proof of Theorem 3.3. Let $F$ be a maximum size 2-part Sperner system, and let $b \in \mathcal{Q}$. Furthermore, let $F(b):=\{a \in \mathcal{P}:(a, b) \in F\}$. Then

$$
\sum_{a \in F(b)} \frac{1}{N_{r(a)}^{(1)}} \leq 1
$$

since, by definition, $F(b)$ is a Sperner system in $\mathcal{P}$. Then

$$
\sum_{b^{\prime} \in \mathcal{Q}_{r(b)}} \sum_{a \in F\left(b^{\prime}\right)} \frac{1}{N_{r(a)}^{(1)}} \frac{1}{N_{r(b)}^{(2)}} \leq 1,
$$


finally taking all possible levels from $\mathcal{Q}$ we have

$$
\sum_{i=0}^{n_{2}} \sum_{b \in \mathcal{Q}_{i}} \sum_{a \in F(b)} \frac{1}{N_{r(a)}^{(1)}} \frac{1}{N_{r(b)}^{(2)}} \leq n_{2}+1 .
$$

This can be rewritten as

$$
\sum_{(a, b) \in F} \frac{1}{N_{r(a)}^{(1)} N_{r(b)}^{(2)}} \leq n_{2}+1,
$$

which is exactly the same, as inequality (3.8). Since $F$ is optimal, therefore, by Lemma 3.7, inequality (3.10) holds with equality, and the same holds for inequality (3.9). (Note that for a regular poset (3.10) follows from (3.1).)

Now Theorem 2.11 implies that for each $b \in \mathcal{Q}$ the family $F(b)$ is a full level in $\mathcal{P}$. If $n_{1}=n_{2}$ then we can repeat our reasoning, exchanging the roles of $\mathcal{P}$ and $\mathcal{Q}$ which clearly finishes the proof. However, if $n_{2}<n_{1}$, then we have to work a little bit more - but the remaining part is about only numbers, therefore the same method what was used in the proof of Theorem 1 in [3] finishes the proof of Theorem 3.3.

At the end of this paper we list now some poset classes for which Theorem 3.3 holds. Those are products of any two posets presented below.

The first four classes of posets listed below are regular and level-connected:

(i) The $n$th power of a star $\mathcal{S}_{k}^{n}$ : the direct product of $n$ copies of a star $\mathcal{S}_{k}$. Note that $\mathcal{S}_{k}^{n}$ can be represented as the set of all $n$-tuples $a^{n} \in$ $\{0,1, \ldots, k\}^{n}$ where the rank of an element $a^{n}$ is defined as the number of nonzero coordinates in $a^{n}$.

(ii) The linear poset (linear lattice) $\mathcal{L}_{n}(q)$ : the poset of all subspaces of a vector space $G F(q)^{n}$ ordered by inclusion.

(iii) The affine poset $\mathcal{A}_{n}(q)$ : the poset of all affine subspaces of a vector space $G F(q)^{n}$ ordered by inclusion. The rank of an element $V$ in both posets is the dimension of $V$.

(iv) Let $\mathcal{P}$ be a ranked poset and let $l, m$ be integers where $0 \leq l<m \leq$ $r(\mathcal{P})$. Then the poset $\mathcal{P}(l, m):=\mathcal{P}_{l} \cup \ldots \cup \mathcal{P}_{m}$ is called a truncated poset of $\mathcal{P}$. This gives another class of regular, unimodal, level-connected posets obtained from $\mathcal{S}_{k}^{n}, \mathcal{L}_{n}(q), \mathcal{A}_{n}(q)$.

(v) The product of $n$ chains $C\left(k_{1}, \ldots, k_{n}\right)$ of sizes $k_{1} \geq \ldots \geq k_{n}$ with $k_{2}+\ldots+k_{n} \geq k_{1}$ is an example of a class of strictly normal unimodal posets, which are not regular (see [13]).

For other classes of strictly normal unimodal posets see [8] (Chapter 4.6). 


\section{References}

[1] R. Ahlswede, Z. Zhang, An identity in combinatorial extremal theory. Adv. Math. 80 (2) (1990), 137-151.

[2] R. Ahlswede, Z. Zhang, On cloud-antichains and related configurations, discrete Math. 85 (1990), 225-245.

[3] H. Aydinian and P.L. Erdös, All maximum size 2-part Sperner systems - in short, Comb. Prob. Comp. 16 (4) (2007), 553-555.

[4] K.A. Baker, A Generalization of Sperner's Lemma, J. Comb.Theory 6 (1969), 224-225.

[5] B. Bollobás, On generalized graphs, Acta Mathematica Academiae Scientiarum Hungaricae 16 (1965) (34), 447-452.

[6] K. Engel, Strong properties in partially ordered sets, I. Discrete Math. 47 (1983), 229-234.

[7] K. Engel, Strong properties in partially ordered sets, II. Discrete Math. 48 (1984), 187-196.

[8] K. Engel, Sperner Theory, Encyclopedia of Mathematics and its Applications, Vol 65, Cambridge Univ. Press, 1997.

[9] Paul Erdös, On a lemma of Littlewood and Offord, Bull. of the Amer. Math. Soc. 51 (1945), 898-902.

[10] P.L. Erdős and G.O.H. Katona, Convex hulls of more-part Sperner families, Graphs and Combin. 2 (1986), 123-134.

[11] P.L. Erdős and G.O.H. Katona, All maximum 2-part Sperner families, J. Combin. Theory (A) 43 (1986), 58-69.

[12] R.L. Graham and L.H. Harper: Some results on matchings in bipartite graphs, SIAM J. Appl. Math 17 (1969), 1017-1022.

[13] J.D. Griggs: Maximum antichains in the product of chains. Order 1 (1984), no. 1, 21-28.

[14] G.O.H. Katona: On a conjecture of Erdős and a stronger form of Sperner's theorem, Studia Sci. Math. Hungar. 1 (1966), 59-63.

[15] G.O.H. Katona, A generalization of some generalizations of Sperner's Theorem, J. Combin. Theory (B) 12 (1972), 72-81.

[16] D.J. Kleitman, On a lemma of Littlewood and Offord on the distribution of certain sums, Math. Zeit. 90 (1965), 251-259. 
[17] D.J. Kleitman, On an extremal property of antichains in partial orders. The LYM property and some of its implications and applications, in Combinatorics, Part 2: Graph theory; foundations, partitions and combinatorial geometry, Math. Centre Tracts, No. 56, Math. Centrum, Amsterdam, (1974), 77-90.

[18] L. Lovász, Combinatorial Problems and Exercises, 2nd ed., NorthHolland, Amsterdam, 1993. Problem 13.21 (b)

[19] D. Lubell, A short proof of Sperner's lemma, J. Comb. Theory 1 (1966) (2), 299.

[20] L.D. Meshalkin, Generalization of Sperner's theorem on the number of subsets of a finite set, Theory of Probability and its Applications 8 (1963) (2), 203-204.

[21] A. Sali, A note on convex hulls of more-part Sperner families, J. Comb. Th. (A) 49 (1988), 188-190.

[22] J. Schonheim, A generalization of results of P. Erdős, G. Katona, and D.J. Kleitman concerning Sperner's theorem, J. Comb. Theory (A) 11 (1971), 111-117.

[23] E. Sperner, Ein Satz über Untermengen einer endlichen Menge, Math. Z. 27 (1928), 544-548.

[24] K. Yamamoto, Logarithmic order of free distributive lattice, J. Math. Soc. Japan 6 (1954), 343-353. 